\title{
Application of Lie-Group Theory for solving Calogero- Bogoyavlenskii- Schiff Equation
}

\author{
Raj Kumar \\ Department of Mathematics, V.B.S. Purvanchal University Jaunpur-222003, India.
}

\begin{abstract}
This work deals with exact solutions of (2+1)-dimensional Calogero-Bogoyavlenskii-Schiff (CBS) equation. Solutions so obtained are derived by using similarity transformations method via Lie-group theory. Exploiting Lie-symmetries and using invariance property, the number of independent variables is reduced by one by using the method, which turns the CBS equation into a new ordinary differential equation. Thereafter, Author attained the explicit solution of the system under the restrictions imposed upon the functions and arbitrary constants involved in the infinitesimals which are derived by the STM. The importance of analytical solution increased since their physical analysis is described on the basis of graphical representation of profiles with the help of numerical simulations.
\end{abstract}

Keywords: CBS equation. Similarity transformations method. Similarity variables. Exact solutions

\section{Introduction}

Several nonlinear evolution equations (NLEEs) occur in various fields of mathematical and physical sciences as well as in Engineering like biology, fluid dynamics, quantum and plasma physics, thermal engineering and optics [1-5] etc. To understand the complex phenomena of NLEEs, it is quite demanding to get its closed form solutions since such solutions are much helpful to analyze directly the physical nature of the systems. Author added his contribution in the same direction since he is seeking the closed form solution of the following $(2+1)$ dimensional Calogero-Bogoyavlenskii-Schiff (CBS) equation

$u_{x t}+u_{x x x z}+4 u_{x} u_{x z}+2 u_{x x} u_{z}=0$

where $u=u(x, z, t)$ is used for brevity. In the article, subscript occurring with a term denotes its partial derivative with respect to the subscript variable. In Eq. (1.1), $u_{t}$ describes the time evolution of the wave, while the terms $u_{x x} u_{z}$ and $u_{x} u_{x z}$ etc. account for nonlinearity of the wave.

The CBS equation has some physical oriented history like it can be written as potential form [6]. The CBS equation was initially constructed by Bogoyavlenskii and Schiff in different ways [7-10]. Recent history of some past researches show that several effective methods for obtaining exact solutions of the CBS equation are contributed by a diverse group of researchers across the globe [9-14], for example, the periodic and soliton solutions of the CBS equation were obtained by Gandarias et al. [11]. Its integrability has been proved by Zhang et al.[12] and derived also the symmetry reductions of the equation. Li and Chen [13] found the exact solutions by using the generalized Raccati equation expansion method. Wang and Yang [14] employed the Hirota Bilinear method for construction of the quasi-periodic wave solutions in terms of theta functions for a Hirota bilinear equation. Finally, the result is applied to the CBS equation.

The researches [7-14] motivate the author to solve Eq. (1.1) analytically by using the similarity transformations method (STM). The fundamental idea behind the method is to reduce the number of independent variables by one in the PDE. The reduction of variables occurs exploiting the symmetries and using invariance property of the PDE under Lie-group theory. The theory and its applications can be grasped by the researches and text books [15-20]. Once the PDE reduces into another PDE with lesser number of independent variable, one can obtain semi analytical/analytical solution of the PDE. On getting analytical solutions, it is easier to analysis physically to the PDE.

\section{Exact Solutions}

In this section, author explained briefly all the steps of the STM method to keep the work self-confined. The detailed description of the method and their applications may be studied in the literature [1-4, 15-20] and references therein.

Author considered the one--parameter ( $\epsilon)$ Lie group of infinitesimal transformations in $(x, z, t, u)$ which are given by

$x^{*}=x+\epsilon \xi^{(1)}(x, z, t, u)+O\left(\epsilon^{2}\right)$,

$z^{*}=z+\epsilon \xi^{(2)}(x, z, t, u)+O\left(\epsilon^{2}\right)$,

$t^{*}=t+\epsilon \tau(x, z, t, u)+O\left(\epsilon^{2}\right)$, 
$u^{*}=u+\epsilon \eta(x, z, t, u)+O\left(\epsilon^{2}\right)$,

where $\epsilon$ is a parameter and $\xi^{(1)}, \xi^{(2)}, \tau, \eta$ are corresponding to the variables $x, z, t$ and $u$ respectively. Using invariance property, the following infinitesimals can be found after performing rather tedious calculations which can be done by hand or using any symbolic program in Maple

$\xi^{(1)}=F_{1}(t)+\frac{1}{4}\left(\mathrm{c}_{1} \mathrm{t}+2 \mathrm{c}_{2}-2 \mathrm{c}_{4}\right) x$,

$\xi^{(2)}=\frac{1}{2} c_{1} z t+c_{6} t+c_{4} z+c_{5}$,

$\tau=\frac{1}{2} \mathrm{c}_{1} \mathrm{t}^{2}+\mathrm{c}_{2} \mathrm{t}+\mathrm{c}_{3}$,

$\eta=-\frac{1}{2} \mathrm{~F}_{1}^{\prime}(\mathrm{t}) \mathrm{z}+\mathrm{F}_{2}(\mathrm{t})+\frac{1}{8}\left(-2 \mathrm{c}_{1} \mathrm{t}-4 \mathrm{c}_{2}+2 \mathrm{c}_{4}\right) \mathrm{u}+\frac{1}{8} \mathrm{c}_{1} \mathrm{xz}+\frac{1}{4} \mathrm{c}_{6} \mathrm{x}$,

where $c_{\mathrm{i}}^{\prime} \mathrm{s}$ are arbitrary parameters and $F_{1}(t), F_{2}(t)$ are arbitrary functions.

Similarity solution of the CBS equation is obtained by choosing the appropriate choice of arbitrary constants and regular arbitrary functions $F_{1}(t)$ and $F_{2}(t)$ so that it can be solved in explicit form. Since, the explicit solution is quite helpful to explain physically meaningful solution on the basis of its graphical representation. Further, author have taken the choice of arbitrary function $F_{1}(t)=A$ while $F_{2}(t)$ is arbitrary, then adjusting the parameters as $\mathrm{c}_{2} \neq \mathrm{c}_{4} \neq 0, c_{1}=c_{3}=c_{5}=0$. Thus to get the exact solution of the CBS equation (1.1), the corresponding characteristic equation is

$\frac{d x}{\xi^{(1)}(x, z, t, u)}=\frac{d z}{\xi^{(2)}(x, z, t, u)}=\frac{d t}{\tau(x, z, t, u)}=\frac{d u}{\eta(x, z, t, u)}$

Inserting infinitesimals to get similarity form of $u$ for the Eq. (1.1)

$\frac{d x}{F_{1}(t)+\frac{1}{2}\left(c_{2}-c_{4}\right) x}=\frac{d z}{c_{4} z}=\frac{d t}{c_{2} t}=\frac{d u}{F_{2}(t)+\frac{1}{2}\left(-c_{2}+c_{4}\right) u}$

The similarity form of the solution of Eq. (1.1) can be written as

$u=\frac{t^{\frac{a-1}{2}}}{c_{2}} \int \frac{F_{2}(t)}{t^{\frac{a+1}{2}}} d t+\frac{1}{t^{\frac{a-1}{2}}} F(X, Z)$,

where $X=t^{\frac{a-1}{2}}\left(-\frac{2 A}{c_{2}(a-1)}\right) x$ and $Z=\frac{z}{t^{a}}$ are similarity variables, $\mathrm{F}$ is similarity function and new introduced arbitrary constant is given by $a=\frac{c_{4}}{c_{2}}$.

Inserting the value of $u$ from Eq. (2.5) into (1.1), we get

$\frac{a-1}{2} X F_{X X}-a Z F_{X Z}+(a-1) F_{X}+F_{X X X Z}+4 F_{X} F_{X Z}+2 F_{X X} F_{Z}=0$.

Since the PDE (2.6) is nonlinear and it has two independent and one dependent variable, so again applying STM on (2.6) provides the following infinitesimals

$\overline{\xi^{(1)}}=-\frac{k_{1} X}{2}+k_{2}$

$\overline{\xi^{(2)}}=k_{1} Z$,

$\bar{\eta}=\frac{k_{1} F}{2}-(a-1) k_{2} \frac{Z}{4}+k_{3}$

where $\mathrm{k}_{\mathrm{i}}^{\prime} \mathrm{s}$ are arbitrary constants.

Therefore, the corresponding Lagrange's system for (2.6) is

$\frac{d X}{-\frac{k_{1} X}{2}+k_{2}}=\frac{d Z}{k_{1} Z}=\frac{d F}{\frac{k_{1} F}{2}-(a-1) k_{2} \frac{Z}{4}+k_{3}}$.

Treating $\mathrm{k}_{1} \neq 0, B=\frac{k_{2}}{k_{1}}$ and $\mathrm{C}=-\frac{2 k_{3}}{k_{1}}$, then similarity transformation predicts the following form of the function

$F$ for the Eq. (2.6)

$F=B(1-a) Z+Z^{\frac{1}{2}} G\left(X_{1}\right)+c$,

with similarity variable, $X_{1}=(X-4 B) \mathrm{Z}^{\frac{1}{2}}$.

It reduces to Eq. (2.6) as follows

$X_{1} G^{\prime \prime \prime \prime}+4 G^{\prime \prime \prime}+6 X_{1} G^{\prime} G^{\prime \prime}-X_{1} G^{\prime \prime}+2 G G^{\prime}+8\left(G^{\prime}\right)^{2}-2 G^{\prime}=0$,

where prime denotes the derivative of $G$ with respect to $X_{1}$.

Eq. (2.10) can be satisfied by

$G=\frac{X_{1}}{4}+\mathrm{B}_{1}$,

where $B_{1}$ is an arbitrary constant of integration. Consequently the solution of CBS Eq. (1.1) can be furnished by

$$
\begin{aligned}
u(x, z, t) & =\frac{t^{t-1 / 2}}{c_{2}}+\frac{C}{t^{(1-a) / 2}}+\frac{B(1-a)}{t^{(1+a) / 2}} z+\frac{z}{4 t}\left(x t^{(a-1) / 2}-2 A \frac{t^{(a-1) / 2}}{c_{2}(a-1)}-4 B+B_{1}\right) \\
& +\int \frac{F_{2}(t)}{t^{\frac{a+1}{2}}} d t .
\end{aligned}
$$




\section{Analysis and Discussions}

A similarity solution has been found explicitly in terms of $x, z$ and $t$ for CBS equation which is different from previous findings [9-14]. The Eq. (2.12) is explicit solution of the problem (1.1). Since the solutions involve arbitrary constants $\mathrm{k}_{1}, \mathrm{k}_{2}$ etc., as well as function $F_{2}(t)$, therefore evolutionary profile can be observed via Fig. 1 at $t=0.5$ and $t=5.28$ for all $-10 \leq \mathrm{x}, \mathrm{y} \leq 10$. Physical structure of the quantities $u$ show elasticity in amplitude of the wave. Author have set $c_{2}=1.9063, \mathrm{k}_{1}=1.8797, \mathrm{k}_{2}=0.8178, \mathrm{k}_{3}=0.4253, \mathrm{~B}_{1}=0.5944, \mathrm{c}_{4}=$ $1.0225, \mathrm{~B}=0.3127, A=0.2607, a=0.5364, a_{-} 4=0.1788, F_{2}(t)=t^{\frac{a+1}{2}} \sin \left(a_{4} \mathrm{t}\right)$ in Fig. 1 . The value of an arbitrary constant is taken as a random number in MATLAB code to get physically meaningful profile of a solution, Author have read that specific value and put here. Thus, analytical solution (2.12) is supplemented by numerical simulation.

\section{Conclusions}

Exact solution of (2+1)-CBS equation is obtained by employing STM successfully which is different from previous findings [10-14]. This work may lead to further research in this important area. As discussed in the above, Lie-group theory is applied to obtain a new closed form solution of the CBS equation and given by the Eq. (2.12). The solutions reflect elastic soliton behaviour of the wave which can be used to test accuracy, comparison and analysis of numerical results in the field. This appears to be more suitable than the previous findings [9-14] as it provides physical analysis of an exact solution of the (2+1)-CBS equation. Again the STM used here can be extended to other exact solutions of NLEEs which are arising in theoretical and applied Physics, Engineering and the like.
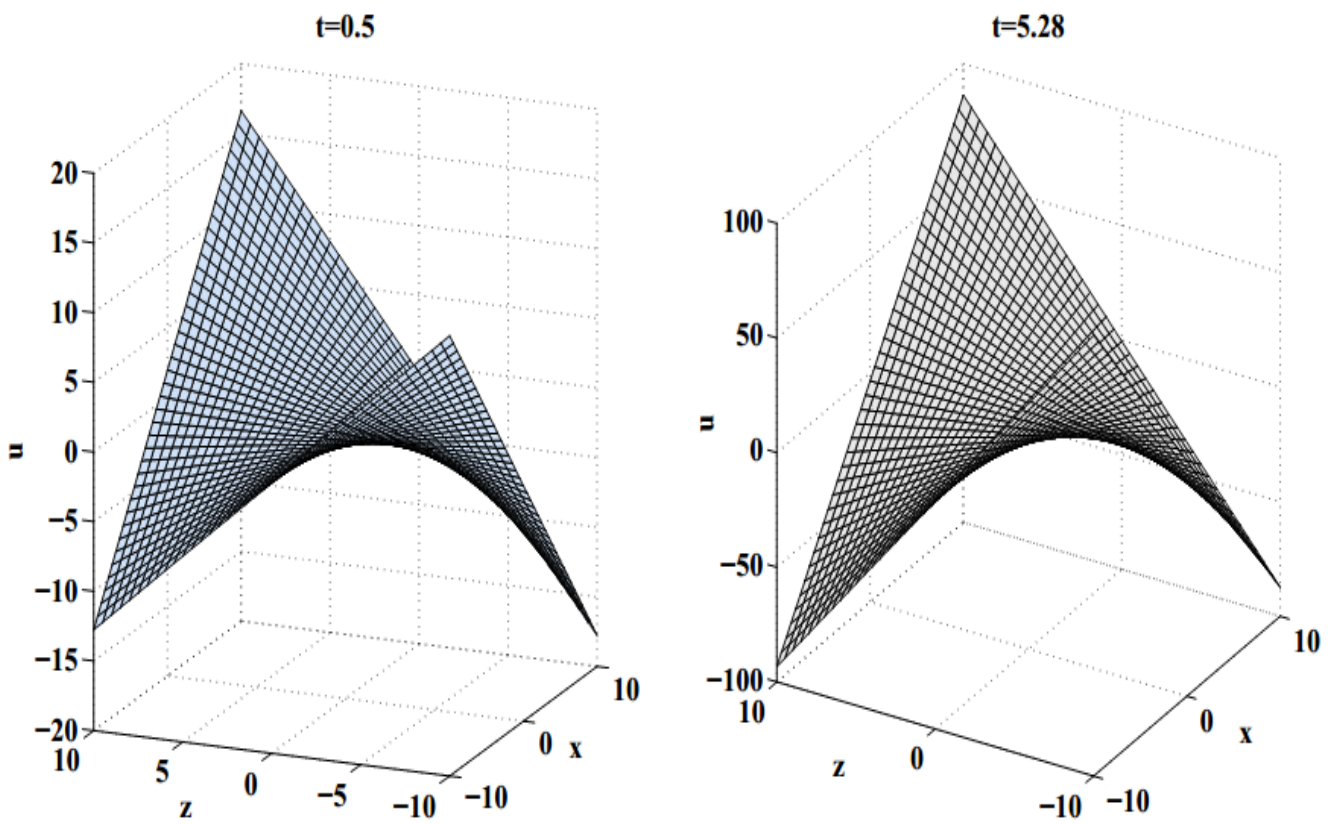

Fig. 1: Variation in $u(x, z, t)$ for (3.12) with varying time at $t=0.5$ and 5.28

\section{References}

[1]. M. Kumar, and R. Kumar, Some more solutions of Burgers' equation. IC-MSQUARE 2014. J. Phys.: Conference Series 574, 2015, 012038. DOI: $10.1088 / 1742-6596 / 574 / 1 / 012038$

[2]. M. Kumar, A. Kumar, and R. Kumar, Similarity solutions of the Konopelchenko-Dubrovsky system using Lie group theory, Computer \& Mathematics with Application, 71(10), 2016, 2051-2059.

[3]. M. Kumar, and R. Kumar, On some new exact solutions of incompressible steady state Navier-Stokes equations, Meccanica 49, 2014, 335-345.

[4]. M. Kumar, and R. Kumar, on new similarity solutions of the Boiti-Leon-Pempinelli system, Communication of Theoretical Physics (Beijing) 61, 2014, 121-126.

[5]. Z. Jiang, and R. K. Bullough, Combined $\bar{\partial}$ and Riemann-Hilbert inverse methods for integrable nonlinear evolution equations in $(2+$ 1)-dimensions, Journal of Physics A: Mathematical and General, 20, 1987, L429-L435.

[6]. O. I. Bogoyavlenskii, Overturning solitons in new two-dimensional integrable equations, Mathematics of the USSR-Izvestiya, 34(2), 1990, 245-259.

[7]. A. M. Wazwaz, New solutions of distinct physical structures to high-dimensional nonlinear evolution equations, Applied Mathematics and Computation, 196, 2008, 363-370.

[8]. Y. Peng, New types of localized coherent structures in the Bogoyavlenskii-Schiff equation, Int. J. Theor. Phys. 45 (9) (2006) 17791783. 
[9]. T. Kobayashi, and K. Toda, The Painlevé test and reducibility to the canonical forms for higher-dimensional soliton equations with variable-coefficients, Symmetry, Inerrability and Geometry: Methods and Applications, 2, 2006, 1-10.

[10]. M. S. Bruzon, M. L. Gandarias, C. Muriel, J. Ramírez, S. Saez, and F. R. Romero, The Calogero-Bogoyavlenskii-Schiff equation in (2 + 1)-dimensions, Theoretical and Mathematical Physics, 137(1), 2003, 1367-1377.

[11]. M. L. Gandarias, and M. S. Bruzon, Symmetry group analysis and similarity solutions of the CBS equation in (2+1)-dimensions, Proceedings of Applied Mathematics and Mechanics, 8, 2008, 10591-10592. DOI 10.1002/pamm.200810591

[12]. H. P. Zhang, Y. Chen, and B. Li, Infinitely many symmetries and symmetry reduction of the (2+1)dimensional generalized CalogeroBogoyavlenskii-Schiff equation, Acta Physica Sinaca, 58, 2009, 7393-7396.

[13]. B. Li, and Y. Chen, Exact analytical solutions of the generalized Calogero-Bogoyavlenskii-Schiff equation using symbolic computation, Czechoslovak Journal of Physics, 54, 2004, 517-528.

[14]. J. Wang, and X. Yang, Quasi-periodic wave solutions for the $(2+1)$-dimensional generalized Calogero-Bogoyavlenskii-Schiff (CBS) equation, Nonlinear Analysis, 75, 2012, 2256-2261.

[15]. M. Kumar, R. Kumar and A. Kumar, Some more similarity solutions of the $(2+1)$-dimensional BLP system, Computers \& Mathematics with Applications, 70, 2015, 212-221.

[16]. M. Kumar, R. Kumar and A. Kumar, On similarity solutions of Zabolotskaya-Khokhlov equation, Computers \& Mathematics with Applications, 68, 2014, 454-463.

[17]. R. Kumar, On Exact Solutions of (2+1)-Dissipative ZK Equation, International Journal of Science, Environment and Technology, 4(5), 2015, 1430-1437.

[18]. R. Kumar, M. Kumar and A. Kumar, Some Soliton Solutions of Non linear Partial Differential Equations by Tan-Cot Method, IOSR Journal of Mathematics, 6, 2013, 23-28.

[19]. G. W. Bluman, and J. D. Cole, Similarity Methods for Differential Equations, (New York, Springer-Verlag, 1974).

[20]. P. J. Olver, Applications of Lie Groups to Differential Equations, (New York, Springer-Verlag, 1993). 